Article

\title{
Microwave-Assisted Classic Ullmann C-C Coupling Polymerization for Acceptor-Acceptor Homopolymers
}

\author{
Zijie Li, Yusheng Chen, Pan Ye, Xiangli Jia, Xiaoxi Wu, Jianfei Wu, Qinqin Shi *, Aidong Peng and \\ Hui Huang * \\ College of Materials Science and Opto-Electronic Technology \& Center of Materials Science and Optoelectronics \\ Engineering \& CAS Center for Excellence in Topological Quantum Computation \& CAS Key Laboratory of \\ Vacuum Physic, University of Chinese Academy of Sciences, Beijing 100049, China; lizijie_win@163.com (Z.L.); \\ chenyusheng767@gmail.com (Y.C.); yepan1108@163.com (P.Y.); jiaxiangli14@mails.ucas.edu.cn (X.J.); \\ estherwuxiaoxi@gmail.com (X.W.); wujianfeidbj@gmail.com (J.W.); paidong@ucas.ac.cn (A.P.) \\ * Correspondence: shiqinqin@ucas.edu.cn (Q.S.); huihuang@ucas.ac.cn (H.H.); \\ Tel.: +86-010-6967-1743 (Q.S); +86-010-6967-1736 (H.H.)
}

Received: 16 September 2019; Accepted: 19 October 2019; Published: 24 October 2019

\begin{abstract}
Developing cheap, clean and atomic-efficient synthetic methodologies for conjugated polymers are always critical for the field of organic electronics. Herein, classic Ullmann coupling polymerization is developed to synthesize a series of Acceptor-Acceptor (A-A) type homopolymers with microwave-assistance, which are supported by nuclear magnetic resonance (NMR), matrix-assisted laser desorption/ionization time of flight mass spectrometry (MALDI-TOF), elemental analysis (EA) and gel permeation chromatography (GPC). The physicochemical properties of these polymers are studied by UV-vis spectroscopy, cyclic voltammetry $(\mathrm{CV})$, thermal gravimetric analysis (TGA), and density functional theory (DFT) calculation. Furthermore, these A-A homopolymers are used as acceptors for all-polymer solar cells (All-PSCs), affording a promising efficiency of 3.08\%, which is the highest value for A-A-homopolymer-based organic solar cells.
\end{abstract}

Keywords: A-A homopolymers; classic Ullmann coupling polymerization; microwave-assistance; organic solar cells

\section{Introduction}

Polymeric semiconductors are emerging as crucial components for state-of-the-art opto-electronic technologies, including organic thin film transistors (TFTs), organic photovoltaics (OPV) and organic photodetectors (OPDs), due to their tunable energy levels, potentially low cost manufacturing, and solution-processability [1-4]. To tune the energy levels and enhance intramolecular charge transfer, the donor-acceptor (D-A) strategy has been used to construct conjugated polymers by various transition metal-mediated aryl coupling methodologies, including Stille coupling and Suzuki coupling reactions [5-12]. Although D-A type copolymers have demonstrated great success in the area of organic electronics, they still suffered from complicated structures and costly synthetic routes. In comparison, homopolymers demonstrated a promising future for commercialization due to their simple structures, easily synthetic routes, and potentially low-cost manufacture [13]. For example, poly(3-hexylthiophene) (P3HT), as one of the Donor-Donor (D-D) conjugated polymers available in quantities over $10 \mathrm{~kg}$, was considered as a promising material for commercial electro-devices [14]. In addition, D-D homopolymer poly-4,8-bis-(5-((2-ethylhexyl)thio)thiophen-2-yl)benzo[1,2-b:4,5-b']-dithiophene (PBDTT-S) based on benzo[1,2-b:4,5- $\left.\mathrm{b}^{\prime}\right]$ dithiophene (BDT) units was reported for OPVs with a high power conversion efficiency (PCE) of 7.05\% [13]. On the other hand, acceptor-acceptor (A-A) homopolymers are relatively rare, although they exhibit excellent electronic affinities and air-stability 
for high-performance organic electronics [15-19]. For example, Guo and coworkers synthesized an A-A homopolymer, poly (2,2'-bithiazolothienyl-4,4',10,10'-tetracarboxydiimide) (PDTzTI) through Stille coupling for organic field effect transistors (OFETs) devices, which exhibits a remarkable electron mobility $\left(\mu_{\mathrm{e}}\right)$ of $1.61 \mathrm{~cm}^{2} \mathrm{~V}^{-1} \mathrm{~s}^{-1}$ [20]. Reynolds et al. employed an isoindigo unit to synthesize poly(isoindigo) through Suzuki polycondensation, which was used as an acceptor for OPVs, affording a relatively low efficiency of $0.47 \%$ [21]. Unfortunately, these synthetic methods usually involve several synthetic steps and toxic reagents (e.g., tin), which is detrimental for commercializing organic electronic technologies. Thus, it is critical to develop cheap, clean and atomic-efficient synthetic methodologies for high-performance A-A homopolymers.

The classic Ullmann coupling reaction is a copper-mediated methodology used to build conjugated systems upon coupling of two molecules of aryl halides with $\mathrm{C}-\mathrm{C}$ bond formation; its advantages include cheap reagents, straightforward routes, and environmentally friendly oxidants (air or oxygen) [22-24]. Thus, Ullmann coupling has been developed to construct molecular and polymeric conjugated materials for opto-electronics [25,26], medicines [27,28], and dyes [29]. For example, Gorgun et al. [25,26] employed Ullmann coupling to synthesize carbazole-based organic semiconductors for photodiodes and photodetectors with respectable performances. Wang and coworkers [27] used CuI as a catalyst to promote a domino reaction, where the condensation and cyclization reaction were followed by an intramolecular Ullmann type reaction, to give 16H-dibenzo[2,3:6,7][1,4] oxazepino[5,4-b]quinazolin-16-ones in good yields. However, the Ullmann coupling reaction has never been employed to synthesize A-A homopolymers.

In this contribution, classic Ullmann polycondensation was employed to synthesize three A-A homopolymers (PPDI(H), PPDI(OD), and PNDI(OD)) with microwave-assistance, which were supported by nuclear magnetic resonance (NMR), elemental analysis (EA), and gel permeation chromatography (GPC). The physicochemical properties of these polymers were investigated by UV-vis spectroscopy, cyclic voltammetry (CV), thermal gravimetric analysis (TGA), and density functional theory (DFT) calculations. Furthermore, these A-A polymers were employed as acceptors for all polymer solar cells (All-PSCs), affording a promising efficiency of 3.08\%, which is the highest value for A-A homopolymer-based organic solar cells (OSCs).

\section{Results and Discussion}

\subsection{Ullmann Polymerization}

Scheme 1 shows the synthetic route of poly[5,12-dimethyl-2,9-di(tridecan-7-yl)anthra[2,1,9-def:6,5, $10-\mathrm{d}^{\prime} \mathrm{e}^{\prime} \mathrm{f}^{\prime}$ ]diisoquinoline-1,3,8,10(2H,9H)-tetraone] (PPDI(H)). The reaction conditions of Ullmann polymerization are listed in Table 1 . In a typical reaction, $\mathrm{PDI}(\mathrm{H})$ was heated with $40 \%$ equiv. of cuprous iodide and 2 equiv. of base in a solvent. According to the literature [30], polar solvents are good choices for Ullmann polymerization in most instances. Thus, dimethyl sulfoxide (DMSO) was first chosen as the solvent for the polymerization at $80^{\circ} \mathrm{C}$ (Entry 1). However, no product was obtained due to the poor solubility of 2Br-PDI in DMSO. Thus, toluene, another polar and excellent solvent for 2Br-PDI, [31] was used for the reaction (Entry 2), but no product was obtained at $80^{\circ} \mathrm{C}$. Considering temperature is a key parameter for catalytic reaction, when the temperature was raised to $150^{\circ} \mathrm{C}$, a good amount of oligomers with up to 5 repeating units were achieved after $12 \mathrm{~h}$, confirmed by matrix-assisted laser desorption ionization time-of-flight (MALDI-TOF) (Entry 4, Figure S1). Afterwards, the reaction time was extended to $24 \mathrm{~h}$ to afford a higher yield and molecular weight (Entry 5, Figure S2). However, the molecular weight is still too low to be defined as a polymer. 


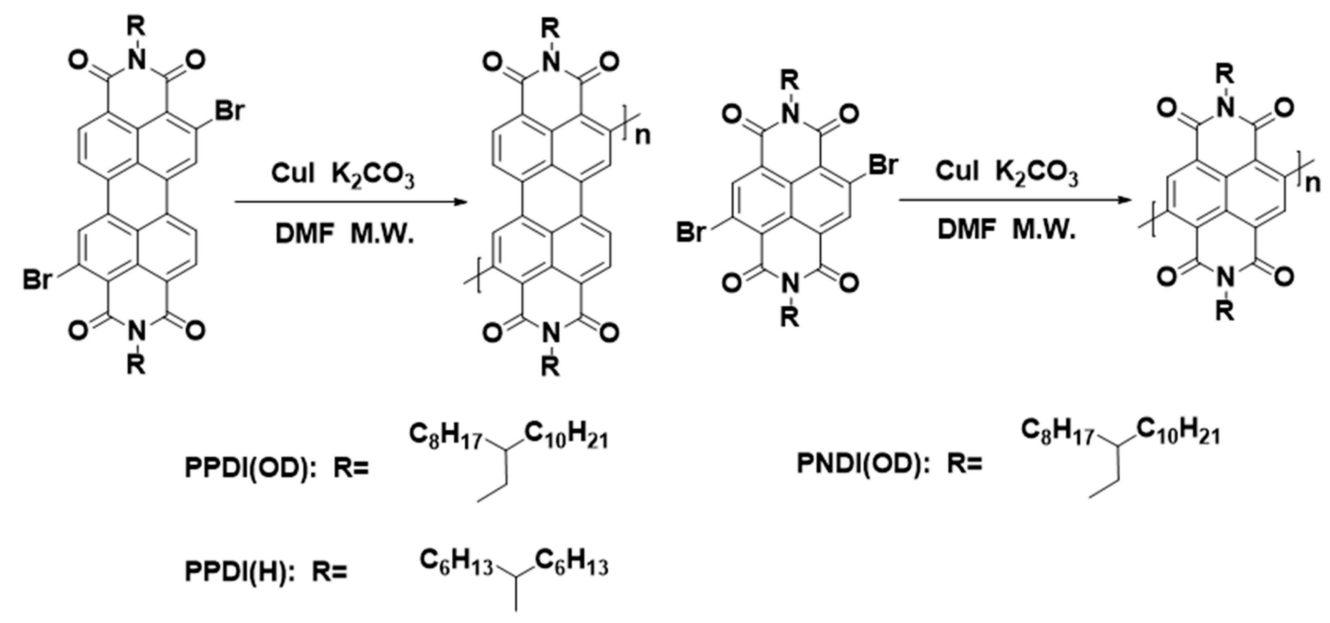

Scheme 1. Synthetic routes of A-A homopolymers.

The microwave-assistance technique has been used as an important tool for coupling polymerization [32,33]. Thus, this technique was employed to improve the efficiency of this Ullmann polymerization. Impressively, the repeating units were significantly improved to 11 units under microwave assistance (Entry 6, Figure S3), while the reaction time was dramatically reduced to $5 \mathrm{~h}$. The number-average molecular weight (Mn) and polydispersity (PDI) are $7.4 \mathrm{kDa}$ and 1.86, respectively, indicating that a polymeric material was achieved.

Table 1. Optimization of the Ullmann polymerization of 2Br-PDI(H).

\begin{tabular}{ccccccc}
\hline Entry $^{\mathbf{a}}$ & Solvent & ${\boldsymbol{T ~}\left({ }^{\circ} \mathbf{C}\right)}$ & Time (h) & Heat & Yield (\%) & Repeat Unit $^{\mathbf{b}}$ \\
\hline 1 & DMSO & 80 & 12 & Oil Bath & 0 & No \\
2 & Toluene & 80 & 12 & Oil Bath & 0 & No \\
3 & DMF & 80 & 12 & Oil Bath & 0 & No \\
4 & DMF & 150 & 12 & Oil Bath & 30 & 5 \\
5 & DMF & 150 & 24 & Oil Bath & 32 & 6 \\
6 & DMF & 150 & 5 & M. W. & 38 & 11
\end{tabular}

a All polymerization reactions used $\mathrm{CuI}$ as a catalyst and $\mathrm{K}_{2} \mathrm{CO}_{3}$ as a base; ${ }^{\mathbf{b}}$ Repeated units of products were determined by MALDI-TOF.

In order to investigate the versatility of the Ullmann polymerization methodology for A-A homopolymers, another two polymers PPDI(OD) and PNDI(OD) were successfully synthesized under the optimized reaction conditions. All three polymers were fully characterized by ${ }^{1} \mathrm{H}-\mathrm{NMR}$, elementary analysis and GPC (Supporting Information, Figures S4-S9). According to the GPC results, the Mn and PDI of PPDI(OD) and PNDI(OD) are $12.8 \mathrm{kDa}$ and 1.84 , and $5.9 \mathrm{kDa}$ and 1.86 , respectively. These respectable Mn and PDI values suggest that versatile applications exist for the use of Ullmann coupling polymerization for synthesizing A-A conjugated polymers. Moreover, these three polymers are soluble in common solvents such as chloroform (CF), chlorobenzene (CB), tetrahydrofuran (THF) and hexane at room temperature. Thermal properties of the polymers were investigated by TGA. As shown in Figure S10, the onset temperatures at 5\% weight loss for these polymers are over $280^{\circ} \mathrm{C}$, which indicated the excellent thermal stability. Moreover, differential scanning calorimetry (DSC) measurement (Figure S11) shows that all these polymers exhibit no obvious melting transition temperature, which indicated the relatively poor crystallinity due to the twisted conformation.

\subsection{Optical and Electrochemical Properties}

UV-vis optical absorption spectra and CV spectrum were performed to investigate the energy levels of PPDI(H), PPDI(OD) and PNDI(OD). The spectra are shown in Figure 1 and Figure S12, 
respectively and the data are listed in Table 2. In solution, both PPDI(H) and PPDI(OD) showed broad absorption bands with maximum absorption peaks at $549 \mathrm{~nm}$ and $546 \mathrm{~nm}$, respectively. The similar absorption profiles indicate turning alkyl chain barely affect the optical properties. Unlike PDI-based homopolymers, the PNDI(OD) showed two absorption bands. The smaller one corresponds to the $\pi-\pi^{*}$ transition, while the larger peak is ascribed to the intramolecular charge transfer (ICT), suggesting the weak ICT process in PNDI(OD). In thin films, PPDI(OD) shows a maximum absorption peak at $545 \mathrm{~nm}$, which is slightly red shifted $(13 \mathrm{~nm})$ in comparison with that PPDI(H), indicating the stereo-demanding alkyl chain may influence the intermolecular packing and thus the optical properties. Also, PNDI(OD) demonstrates two absorption peaks at $368 \mathrm{~nm}$ and $531 \mathrm{~nm}$ in film. Notably, these values are close to those of solutions, suggesting very weak intermolecular interactions. According to the onset of the long wavelength absorption, the optical band gaps $\left(E_{\mathrm{g}}\right)$ of PPDI(H) and PPDI(OD) and PNDI(OD) were estimated to be $1.94 \mathrm{eV}, 1.87 \mathrm{eV}$ and $2.16 \mathrm{eV}$, respectively. According to the $\mathrm{CV}$ measurement, the LUMO energy levels of PPDI(H) and PPDI(OD) and PNDI(OD) were estimated to be $-4.00 \mathrm{eV}$, $-4.05 \mathrm{eV}$ and $-3.84 \mathrm{eV}$, respectively. Thus, HOMO energy levels of PPDI(H) and PPDI(OD) and PNDI(OD) were calculated to be $-5.94 \mathrm{eV},-5.92 \mathrm{eV}$ and $-6.00 \mathrm{eV}$, respectively.
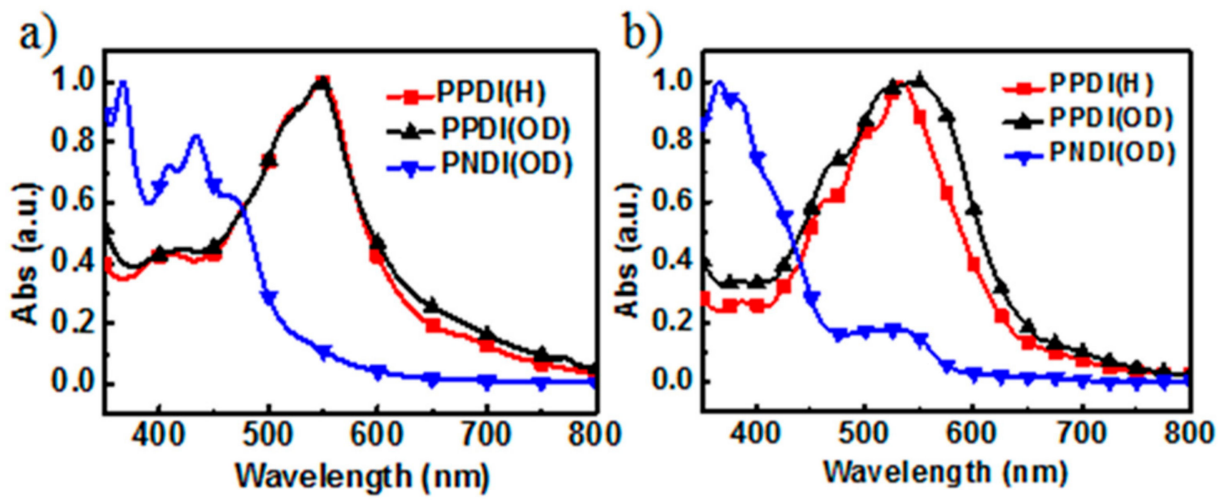

Figure 1. Normalized UV-vis spectra of these polymers in solution (a) and as film (b).

Table 2. Summarized opto-electronic properties of the polymers.

\begin{tabular}{cccccc}
\hline Material & $\lambda_{\text {peak }}{ }^{\mathbf{a}}(\mathbf{n m})$ & $\boldsymbol{\lambda}_{\text {onset }}(\mathbf{e V})$ & $E_{\mathrm{g}}{ }^{\mathbf{b}}(\mathbf{e V})$ & LUMO $(\mathbf{e V})$ & HOMO $^{\mathbf{c}}(\mathbf{e V})$ \\
\hline PPDI(H) & 532 & 638 & 1.94 & -4.00 & -5.94 \\
PPDI(OD) & 545 & 667 & 1.87 & -4.05 & -5.92 \\
PNDI(OD) & 531 & 575 & 2.16 & -3.84 & -6.00 \\
\hline \multicolumn{5}{c}{${ }^{\mathrm{a}}$ all data were measured in films; ${ }^{\mathrm{b}} E_{\mathrm{g}}=1240 / \lambda_{\text {offset }} ;{ }^{\mathrm{c}} \mathrm{HOMO}=\mathrm{LUMO}-E_{\mathrm{g} .}$}
\end{tabular}

\subsection{DFT Calculation}

In order to further understand the optical properties of PPDI and PNDI, DFT calculations were carried out at the level of the B3LYP/6-31G(d, p) as shown in Figure 2 and Figure S13, respectively. From the side view (Figure 2c), dihedral angles of these structures 3-PDI, 4-PDI, and 5-PDI are $55^{\circ}, 56^{\circ}$, and $52^{\circ}$ respectively, indicating the $X$-type structure of PPDI. Obviously, twisted molecules exhibit weak intermolecular interactions and prevent the strong aggregation and large domain size, which may be beneficial for optimal phase separation in the blend films of OSCs [34-37]. The molecular orbital distribution showed that the highest occupied molecular orbital (HOMO) is delocalized along the whole backbone of the molecules, while the lowest unoccupied molecular orbital (LUMO) is localized on the center part of these molecules, indicating moderate intramolecular charge transfer, consistent with the optical absorption. The DFT calculations (Table S2) showed that the energy levels of LUMO/HOMO of these three molecules (3-PDI, 4-PDI, and 5-PDI) are -3.68/-5.95 eV, -3.71/-5.96 eV, $-3.73 /-5.96 \mathrm{eV}$, respectively. Obviously, as the number of repeating units (PDI) increased, energy level of LUMO decreases but HOMO remains unchanged, which thus can narrow the bandgap. 
a)
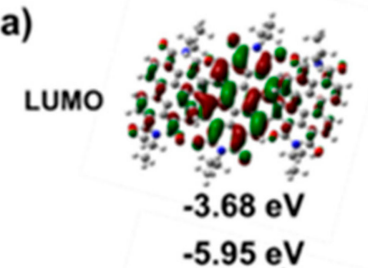

b)

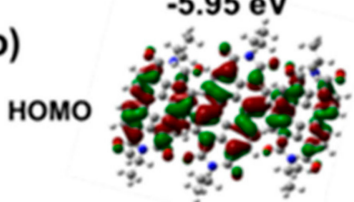

c)

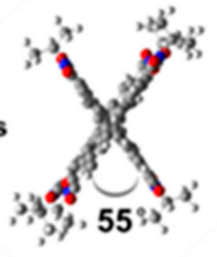

3-PDI

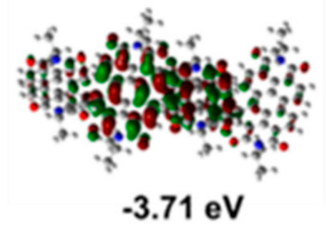

$-5.96 \mathrm{eV}$
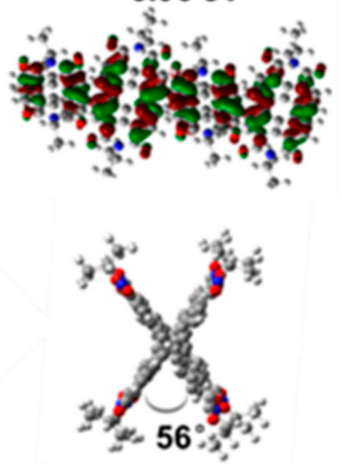

4-PDI

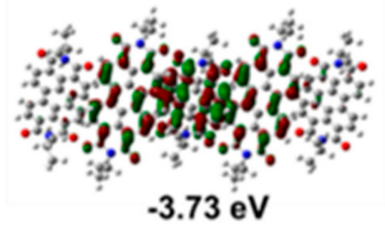

$-5.96 \mathrm{eV}$
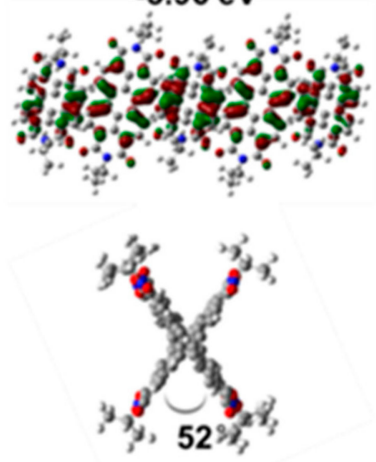

5-PDI

Figure 2. (a,b) Optimized geometry, calculated HOMO and LUMO orbitals of the 3-PDI, 4-PDI and 5-PDI; (c) optimized geometry of three molecules shown in side view.

\subsection{Fabrication and Characterization of All-PSCS}

Among these three A-A homopolymers, $\mathrm{PPDI}(\mathrm{H})$ exhibits complementary absorptions with narrow bandgap materials, such as poly[4,8-bis(5-(2-ethylhexyl)thiophen-2-yl)benzo[1,2-b:4,5- $\left.\mathrm{b}^{\prime}\right]$ dithiophene-co-3-fluorothieno[3,4-b]thiophene-2-carboxylate] (PTB7-Th) and poly[(2,6-(4,8-bis (5-(2-ethylhexyl)thiophen-2-yl)benzo[1,2-b:4,5-b' $]$ dithiophene)-co-(1,3-di(5-thiophene-2-yl)-5,7-bis (2-ethylhexyl)benzo[1,2-c:4,5-c' $]$ dithiophene-4,8-dione)] (PBDB-T) [38,39]. Furthermore, PPDI(H) presents a similar absorption coefficient to these two D-A donor conjugated polymers (Figure S14), suggesting that PPDI $(\mathrm{H})$ may be an excellent acceptor matching with these two donors for All-PSCs.

To investigate the photovoltaic performances of PPDI $(\mathrm{H}) \backslash \mathrm{PTB}$-Th:PPDI $(\mathrm{H})$ and PBDB-T:PPDI(H) based All-PSCs were fabricated with inverted structures of ITO/ZnO/Active layer/MoO3/Ag. The typical current density-voltage $(\mathrm{J}-\mathrm{V})$ curves are shown in Figure $3 \mathrm{a}$ and the photovoltaic parameters were summarized in Table 3. The PTB7-Th:PPDI(H)-based All-PSCs exhibited a promising average PCE of $2.87 \%$, with $V_{O C}$ of $0.58 \mathrm{~V}, J_{S C}$ of $11.24 \mathrm{~mA} \cdot \mathrm{cm}^{-2}$, and $F F$ of 0.450 , and the maximum efficiency is over 3\%, the highest value for A-A homopolymer-based All-PSCs. However, PBDB-T:PPDI(H)-based devices showed a much lower efficiency of $1.95 \%$, with $V_{O C}$ of $0.61 \mathrm{~V}, J_{S C}$ of $7.88 \mathrm{~mA} \cdot \mathrm{cm}^{-2}$, and $F F$ of 0.419. In comparison to PBDB-T-based solar cells, PTB7-Th-based solar cells display lower $V_{O C}$ due to its high-lying HOMO energy level. However, PTB7-Th-based solar cells exhibit relatively higher $J_{S C}$ than PBDB-T-based solar cells, which may be partly ascribed to its better complementary absorption with PTB7-Th. The mobilities of the PTB7-Th/PPDI(H), PTB7-Th/PPDI(OD) and PTB7-Th/PNDI(OD) via the SCLC method (space charge limits current) are shown in Table S3. 
a)

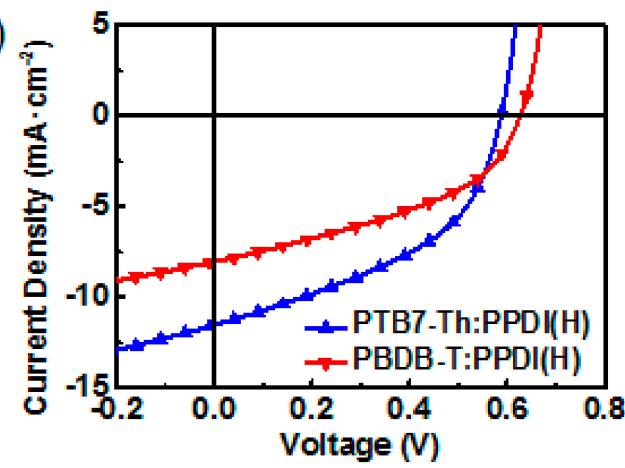

c)

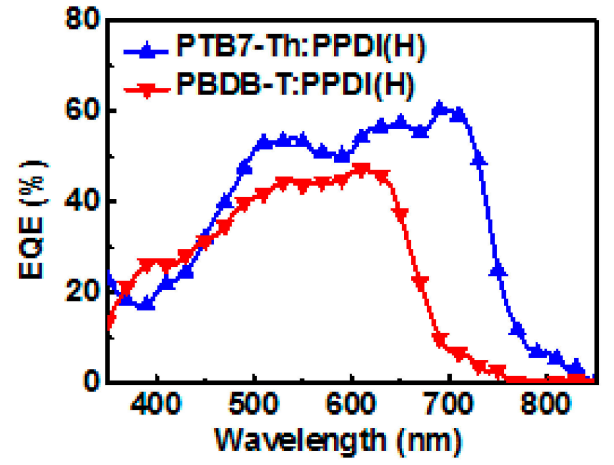

b)

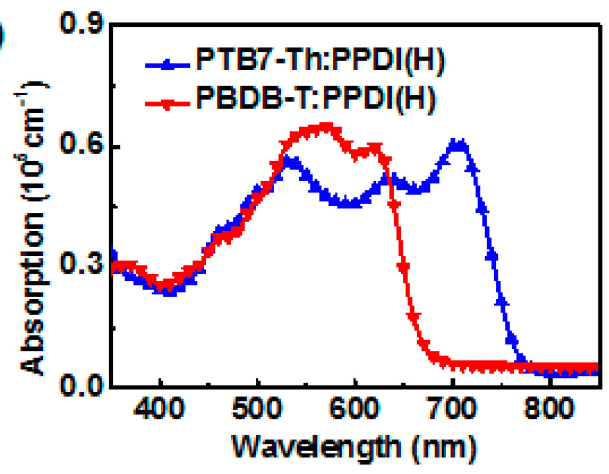

d)

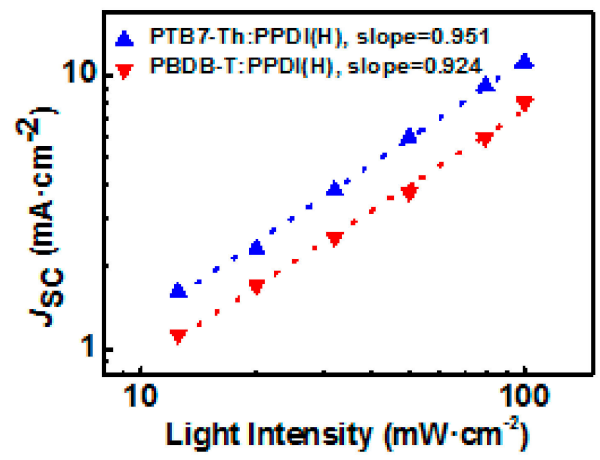

Figure 3. (a) The current density-voltage (J-V) curves of PPDI(H)-based All-PSCs; (b) absorption coefficient of PTB7-Th:PPDI(H) and PBDB-T:PPDI(H) blend films; (c) EQE of PPDI(H)-based All-PSCs; (d) short current density $\left(J_{S C}\right)$ versus light intensity of the PTB7-Th-based devices and PBDB-T-based devices.

Table 3. Photovoltaic properties of All-PSCs under the illumination of AM $1.5 \mathrm{G}, 100 \mathrm{~mW} \cdot \mathrm{cm}^{2}$.

\begin{tabular}{ccccc}
\hline Blend Film & PCE (\%) & $V_{\boldsymbol{O C}}(\mathbf{V})$ & $J_{S C}\left(\mathbf{m A} \cdot \mathbf{c m}^{-\mathbf{2}}\right)$ & $\boldsymbol{F F} \mathbf{~ ( \% )}$ \\
\hline PTB7-Th:PPDI(H) & $2.87 \pm 0.22$ & $0.58 \pm 0.01$ & $11.24 \pm 0.28$ & $45.0 \pm 0.5$ \\
PBDB-T:PPDI(H) & $1.95 \pm 0.13$ & $0.61 \pm 0.01$ & $7.88 \pm 0.24$ & $41.9 \pm 0.4$ \\
\hline
\end{tabular}

To understand the difference of the $J_{S C}$ of these two systems, absorption coefficients of the blend films were measured as shown in Figure 3b. The PBDB-T:PPDI(H) blend films possess stronger light absorption in the range of 550-650 $\mathrm{nm}$ due to the heavily overlapped absorption of these two materials in this area. However, the PTB7-Th:PPDI $(\mathrm{H})$ blend films exhibit a much broader optical absorption in the range of 450-800 $\mathrm{nm}$ due to the narrow bandgap of PTB7-Th in comparison to PBDB-T:PPDI(H) blend films (450-620 nm), which may lead to generation of more excitons and higher $J_{S C}$. The EQE curves of All-PSCs are shown in Figure 3c, which demonstrated that PTB7-Th-based All-PSCs possess broader photoresponse than PBDB-T-based devices, consistent with the optical absorption spectra. Importantly, PTB7-Th: PPDI(H)-based All-PSCs possess stronger photoresponse in the range from 550 to $650 \mathrm{~nm}$, though the blend films absorb less optical light in this area compared to PBDB: PPDI(H) blend films, which may be ascribed to the morphology of the blend films. The exciton split was investigated by the photoluminescence of blend films as shown in Figure 4. Interestingly, the quenching efficiency of PTB7-Th: PPDI(H) blend film is $97.2 \%$, higher than that of PBDB-T: PPDI(H) blend film $(95.6 \%)$, which suggested that electron transfer from the donor polymers to the acceptor polymers in the former system is more efficient than the later one, consistent with the $J_{S C}$ values. Moreover, $J_{S C}$ was measured as a function of illumination intensity for the devices to understand the bimolecular recombination kinetics. In principle, a linear dependence of $\log \left(J_{S C}\right)$ and $\log$ (Plight) with a slope close to 1 suggests weak bimolecular recombination in the photovoltaic devices. As shown in Figure 3d, the slope values for PTB7-Th-based and PBDB-T-based devices are 0.951 and 0.925 respectively, which revealed that the 
bimolecular recombination in PTB7-Th devices is less than that in PBDB-T ones, which may contribute to the larger $J_{S C}$.
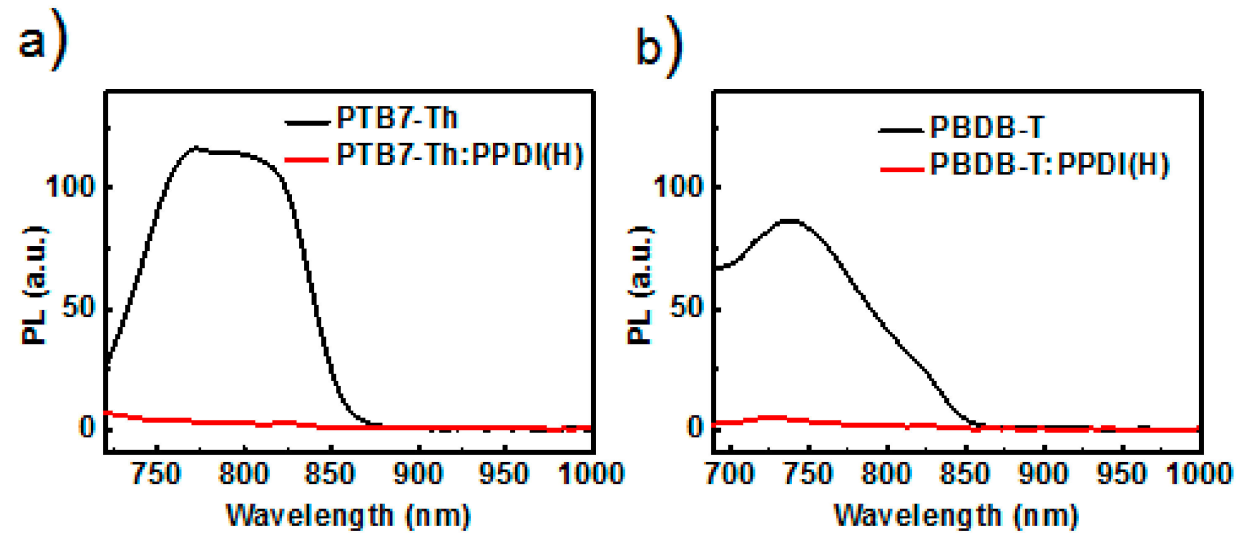

Figure 4. Photoluminescence spectra of PTB7-Th: PPDI(H) blend film (a); and PBDB-T: PPDI $(\mathrm{H})$ blend film (b).

The morphologies of blend films are critical for photovoltaic performance, thus the PTB7-Th: PPDI(H) and PBDB-T: PPDI(H) blend films were investigated by transmission electron microscopy (TEM) and atomic force microscopy (AFM). The TEM images (Figure 5a,b) obviously show that PTB7-Th:PPDI $(\mathrm{H})$ blend films exhibit featured domain size and suitable phase separation, which facilitates exciton diffusion, separation and charge transport [40]. In comparison, PBDB-T: PPDI(H) blend films demonstrate unfeatured morphologies and small domains, which are unfavorable for charge transport. On the other hand, the AFM phase images (Figure 5c,d) exhibit that the root-mean-square (RMS) roughness of PTB7-Th-based blend film and PBDB-T blend film are $0.918 \mathrm{~nm}$ and $1.256 \mathrm{~nm}$, respectively, suggesting both films possess smooth morphologies, beneficial for the contact between blend films and interfacial layers. The powder X-ray diffraction pattern of the PPDI $(\mathrm{H})$ is shown in Figure S15, which shows that no obvious diffraction peaks including crystalline or $\pi-\pi$ stacking were observed.
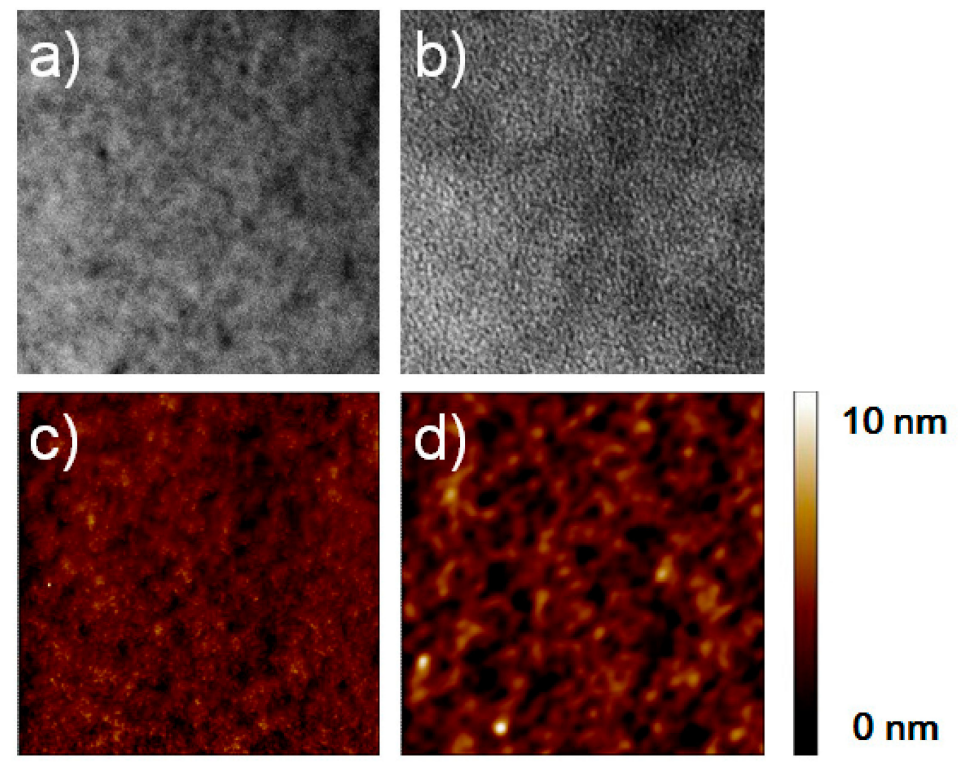

Figure 5. (a,b) TEM images of PTB7-Th: PPDI $(\mathrm{H})$ blend film and PBDB-T: PPDI $(\mathrm{H})$ blend films (the scan size is $10 \mu \mathrm{m} \times 10 \mu \mathrm{m})$; (c,d) AFM phase images of PTB7-Th: PPDI (H) blend film and PBDB-T: $\mathrm{PPDI}(\mathrm{H})$ blend films (scan size is $10 \mu \mathrm{m} \times 10 \mu \mathrm{m}$ ). 


\section{Conclusions}

In summary, we successfully developed Ullmann coupling polymerization as a feasible methodology to synthesize A-A type conjugated homopolymers. Upon optimizing the reaction conditions, three A-A homopolymers with respectable molecular weights were synthesized, which exhibited excellent optical, thermal and electronic properties. In combination with a narrow bandgap donor (PTB7-Th), PPDI(H)-based All-PSCs showed a promising efficiency of 3.09\%, the highest value for A-A homopolymer-based OSCs. This contribution presented an important step towards development of green and cheap tools to synthesize high-performance A-A type conjugated polymers.

Supplementary Materials: The supplementary materials are available online at http://www.mdpi.com/2073-4360/ 11/11/1741/s1.

Author Contributions: Investigation, Z.L., Y.C., P.Y., X.J., X.W. and J.W.; Methodology, Z.L. and Y.C.; Project administration, Q.S., A.P. and H.H.; Supervision, H.H.; Validation, Z.L. and Y.C.; Visualization, Z.L., Y.C. and X.W.; Writing—original draft, Z.L. and Y.C.; Writing—review \& editing, Z.L., Q.S. and H.H.

Funding: The authors acknowledge financial support from the NSFC (21774130), National Key R\&D Program of China (2018FYA 0305800), the Key Research Program of Frontier Sciences, CAS (QYZDB-SSW-JSC046), Key Research Program of the Chinese Academy of Sciences (XDPB08-2), the Strategic Priority Research Program of the Chinese Academy of Sciences (XDB28000000), External Cooperation Programs of the Chinese Academy of Sciences (211211KYSB20170014), Innovation Program of Aerospace Science and Technology of China Aerospace Science and Technology Corporation.

Conflicts of Interest: The authors declare no conflict of interest.

\section{References}

1. Hwang, Y.J.; CourtrightZhou, N.; Dudnik, A.S.; Li, T.I.N.G.; Manley, E.F.; Aldrich, T.J.; Guo, P.; Liao, H.C.; Chen, Z.; Chen, L.X.; et al. All-Polymer Solar Cell Performance Optimized via Systematic Molecular Weight Tuning of both Donor and Acceptor Polymers. J. Am. Chem. Soc. 2016, 138, 1240-1251.

2. Kim, T.; Kim, J.H.; Kang, T.E.; Lee, C.; Kang, H.; Shin, M.; Wang, C.; Ma, B.; Jeong, U.; Kim, T.S. Flexible, Highly Efficient All-Polymer Solar Cells. Nat. Commun. 2015, 6, 8547. [CrossRef] [PubMed]

3. Hwang, Y.J.; Courtright, B.A.E.; Ferreira, A.S.; Tolbert, S.H.; Jenekhe, S.A. 7.7\% Efficient All-Polymer Solar Cells. Adv. Mater. 2015, 27, 4578-4584.

4. Zhou, Y.; Gu, K.L.; Gu, X.; Kurosawa, T.; Yan, H.; Guo, Y.; Koleilat, G.I.; Zhao, D.; Toney, M.F.; Bao, Z. All-Polymer Solar Cells Employing Non-Halogenated Solvent and Additive. Chem. Mater. 2016, 28, 5037-5042. [CrossRef]

5. Ye, L.; Jiao, X.; Zhang, H.; Li, S.; Yao, H.; Ade, H.; Hou, J. 2D-Conjugated Benzodithiophene-Based Polymer Acceptor: Design, Synthesis, Nanomorphology, and Photovoltaic Performance. Macromolecules 2015, 48, 7156-7163. [CrossRef]

6. Jiang, Y.; Lu, L.; Yang, M.; Zhan, C.; Xie, Z.; Verpoort, F.; Xiao, S. Taking the Place of Perylene Diimide: Perylene Tetracarboxylic Tetraester as A Building Block for Polymeric Acceptors to Achieve Higher Open Circuit Voltage in All-Polymer Bulk Heterojunction Solar Cells. Polym. Chem. 2013, 4, 5612-5620. [CrossRef]

7. Liu, S.; Kan, Z.; Thomas, S.; Cruciani, F.; Brédas, J.L.; Beaujuge, P.M. Thieno[3,4-c]Pyrrole-4,6-Dione-3,4-Difluorothiophene Polymer Acceptors for Efficient All-Polymer Bulk Heterojunction Solar Cells. Angew. Chem. Int. Ed. 2016, 55, 12996-13000. [CrossRef]

8. Li, Y.; Yang, Y.; Bao, X.; Qiu, M.; Liu, Z.; Wang, N.; Zhang, G.; Yang, R.; Zhang, D. New $\pi$-Conjugated Polymers as Acceptors Designed for All-Polymer Solar Cells Based on Imide/amide-derivatives. J. Mater. Chem. C. 2016, 4, 185-192. [CrossRef]

9. Liu, S.; Song, X.; Thomas, S.; Kan, Z.; Cruciani, F.; Laquai, F.; Bredas, J.L.; Beaujuge, P.M. Thieno[3,4-c]Pyrrole-4,6-Dione-Based Polymer Acceptors for High Open-Circuit Voltage All-Polymer Solar Cells. Adv. Energy Mater. 2017, 7, 1602574. [CrossRef]

10. Fang, K.; Huang, Y.; Chang, G.; Yang, J.; Shen, Y.; Ye, X. Conjugated Polymers Based on Modified Benzo[1,2-b:4,5- $\mathrm{b}^{\prime}$ ]dithiophene and Perylene Diimide Derivatives: Good Optical Properties and Tunable Electrochemical Performance. Macromol. Res. 2015, 23, 545-551. [CrossRef] 
11. Deng, Z.; Wu, F.; Chen, L.; Chen, Y. Novel Photovoltaic Donor 1-Acceptor-Donor 2-Acceptor Terpolymers with Tunable Energy Levels Based on Difluorinated Benzothiadiazole Acceptor. RSC Adv. 2015, 5, 12087-12093. [CrossRef]

12. Sharma, S.; Kolhe, N.B.; Gupta, V.; Bharti, V.; Sharma, A.; Datt, R.; Chand, S.; Asha, S.K. Improved All-Polymer Solar Cell Performance of n-Type Naphthalene Diimide-Bithiophene P(NDI2OD-T2) Copolymer by Incorporation of Perylene Diimide as Coacceptor. Macromolecules 2016, 49, 8113-8125. [CrossRef]

13. Kim, J.H.; Park, J.B.; Yoon, S.C.; Jung, I.H.; Hwang, D.H. Enhanced and Controllable Open-Circuit Voltage Using 2D-Conjugated Benzodithiophene (BDT) Homopolymers by Alkylthio Substitution. J. Mater. Chem. C. 2016, 4, 2170-2177. [CrossRef]

14. Po, R.; Bernardi, A.; Calabrese, A.; Carbonera, C.; Corso, G.; Pellegrino, A. From Lab to Fab: How Must the Polymer Solar Cell Materials Design Change? An Industrial Perspective. Energy Environ. Sci. 2014, 7 , 925-943. [CrossRef]

15. Gwinner, M.C.; Brenner, T.J.K.; Lee, J.K.; Newby, C.; Ober, C.K.; Mcneill, C.R.; Sirringhaus, H. Organic Field-Effect Transistors and Solar Cells Using Novel High Electron-Affinity Conjugated Copolymers Based on Alkylbenzotriazole and Benzothiadiazole. J. Mater. Chem. 2012, 22, 4436-4439. [CrossRef]

16. Lee, J.K.; Gwinner, M.C.; Berger, R.; Newby, C.; Zentel, R.; Friend, R.H.; Sirringhaus, H.; Ober, C.K. High-Performance Electron-Transporting Polymers Derived from A Heteroaryl Bis(trifluoroborate). J. Am. Chem. Soc. 2011, 133, 9949-9951. [CrossRef] [PubMed]

17. Wang, X.; Lv, L.; Li, L.; Chen, Y.; Zhang, K.; Chen, H.; Dong, H.; Huang, J.; Shen, G.; Yang, Z. High-Performance All-Polymer Photoresponse Devices Based on Acceptor-Acceptor Conjugated Polymers. Adv. Funct. Mater. 2016, 26, 6306-6315. [CrossRef]

18. Ge, C.W.; Mei, C.Y.; Ling, J.; Wang, J.T.; Zhao, F.G.; Liang, L.; Li, H.J.; Xie, Y.S.; Li, W.S.J. Acceptor-Acceptor Conjugated Copolymers Based on Perylenediimide and Benzothiadiazole for All-Polymer Solar Cells. Polym. Sci. Part A Polym. Chem. 2014, 52, 1200-1215. [CrossRef]

19. Banal, J.L.; Subbiah, J.; Graham, H.; Lee, J.K.; Ghiggino, K.P.; Wong, W.W.H. Electron Deficient Conjugated Polymers Based on Benzotriazole. Polym. Chem. 2013, 4, 1077-1083. [CrossRef]

20. Shi, Y.; Guo, H.; Qin, M.; Zhao, J.; Wang, Y.; Wang, H.; Wang, Y.; Facchetti, A.; Lu, X.; Guo, X. Thiazole Imide-Based All-Acceptor Homopolymer: Achieving High-Performance Unipolar Electron Transport in Organic Thin-Film Transistors. Adv. Mater. 2018, 30, 1705745. [CrossRef]

21. Stalder, R.; Mei, J.; Subbiah, J.; Grand, C.; Estrada, L.A.; So, F.; Reynolds, J.R. n-Type Conjugated Polyisoindigos. Macromolecules 2011, 44, 6303-6310. [CrossRef]

22. Matthew, M.D.; Peter, D.K.; Christine, K.L. Synthesis and Characterization of Thiophene-Containing Naphthalene Diimide n-Type Copolymers for OFET Applications. Macromolecules 2010, 43, 6348-6352.

23. Sambiagio, C.; Marsden, S.P.; Blacker, A.J.; McGowan, P.C. Copper Catalysed Ullmann Type Chemistry: From Mechanistic Aspects to Modern Development. Chem. Soc. Rev 2014, 43, 3525-3550. [CrossRef] [PubMed]

24. Bhunia, S.; Pawar, G.G.; Kumar, S.V.; Jiang, Y.; Ma, D. Selected Copper-Based Reactions for C-N, C-O, C-S, and C-C Bond Formation. Angew. Chem. Int. Ed. 2017, 56, 16136-16179. [CrossRef]

25. Gorgun, K.; Caglar, Y.; Yakuphanoglu, F. Synthesis and Photodiode Characterization of Novel Twisted Carbazole Derivatives with 1,3,5-Benzene Core. Silicon 2017, 10, 693-702. [CrossRef]

26. Gorgun, K.; Caglar, Y. Synthesis of Novel Carbazole Derived Substances Using Some Organoboron Compounds by Palladium Catalyzed and Investigation of Its Semiconductor Device Characteristics. J. Mol. Struct. 2018, 1157, 106-111. [CrossRef]

27. Zhang, Y.; Liu, J.Q.; Wang, X.S. An Efficient Synthesis of 16H-Dibenzo[2,3:6,7][1,4] Oxazepino[5,4-b]Quinazolin-16-Ones via An Ullmann Reaction Catalyzed by CuI. Org. Biomol. Chem. 2018, 16, 1679-1685. [CrossRef]

28. Khan, F.; Dlugosch, M.; Liu, X.; Banwell, M.G. The Palladium-Catalyzed Ullmann Cross-Coupling Reaction: A Modern Variant on A Time-Honored Process. Acc. Chem. Res. 2018, 51, 1784-1795. [CrossRef]

29. Zhang, Y.Q.; Qi, L.; Sun, J.P.; Long, J.J. Synthesis of An Anthraquinonoid Disperse Reactive Dye Based on A Ligand-Free Ullmann Reaction. Color. Technol. 2017, 133, 283-292. [CrossRef]

30. Bao, X.; Qiao, X.; Bao, C.; Liu, Y.; Zhao, X.; Lu, Y.; Chen, G. Synthesis of Tamibarotene via Ullmann-Type Coupling. Org. Process Res. Dev. 2017, 21, 748-753. [CrossRef] 
31. Audisio, D.; Messaoudi, S.; Peyrat, J.F.; Brion, J.D.; Alami, M. A General Copper Powder-Catalyzed Ullmann-Type Reaction of 3-Halo-4(1H)-Quinolones with Various Nitrogen-Containing Nucleophiles. J. Org. Chem. 2011, 76, 4995-5005. [CrossRef] [PubMed]

32. Ley, S.V.; Thomas, A.W. Modern Synthetic Methods for Copper-Mediated C(aryl)[bond]O, C(aryl)[bond]N, and C(aryl)[bond]S Bond Formation. Angew. Chem. Int. Ed. 2003, 42, 5400-5449. [CrossRef] [PubMed]

33. Park, Y.S.; Wu, Q.; Nam, C.Y.; Grubbs, R.B. Polymerization of Tellurophene Derivatives by Microwave-Assisted Palladium-Catalyzed Ipso-Arylative Polymerization. Angew. Chem. Int. Ed. 2014, 53, 10691-10695. [CrossRef] [PubMed]

34. Yan, Q.; Zhou, Y.; Zheng, Y.Q.; Pei, J.; Zhao, D. Towards Rational Design of Organic Electron Acceptors for Photovoltaics: A Study Based on Perylenediimide Derivatives. Chem. Sci. 2013, 4, 4389-4394. [CrossRef]

35. Singh, R.; Aluicio-Sarduy, E.; Kan, Z.; Ye, T.; Mackenzie, R.C.I.; Keivanidis, P.E. Fullerene-Free Organic Solar Cells with An Efficiency of 3.7\% Based on A Low-Cost Geometrically Planar Perylene Diimide Monomer. J. Mater. Chem. A 2014, 2, 14348-14353. [CrossRef]

36. Liu, Y.; Mu, C.; Jiang, K.; Zhao, J.; Li, Y.; Zhang, L.; Li, Z.; Lai, J.Y.L.; Hu, H.; Ma, T. A Tetraphenylethylene Core-Based 3D Structure Small Molecular Acceptor Enabling Efficient Non-Fullerene Organic Solar Cells. Adv. Mater. 2015, 27, 1015-1020. [CrossRef]

37. Rajaram, S.; Shivanna, R.; Kandappa, S.K.; Narayan, K.S. Nonplanar Perylene Diimides as Potential Alternatives to Fullerenes in Organic Solar Cells. J. Phys. Chem. Lett. 2012, 3, 2405-2408. [CrossRef]

38. Zhang, S.; Ye, L.; Zhao, W.; Liu, D.; Yao, H.; Hou, J. Side Chain Selection for Designing Highly Efficient Photovoltaic Polymers with 2D-Conjugated Structure. Macromolecules 2014, 47, 4653-4659. [CrossRef]

39. Qian, D.; Ye, L.; Zhang, M.; Liang, Y.; Li, L.; Huang, Y.; Guo, X.; Zhang, S.; Tan, Z.; Hou, J. Design, Application, and Morphology Study of a New Photovoltaic Polymer with Strong Aggregation in Solution State. Macromolecules 2012, 45, 9611-9617. [CrossRef]

40. Brabec, C.J.; Winder, C.; Sariciftci, N.S.; Hummelen, J.C.; Dhanabalan, A.; Van Hal, P.A.; Janssen, R.A.J. A Low-Bandgap Semiconducting Polymer for Photovoltaic Devices and Infrared Emitting Diodes. Adv. Funct. Mater. 2002, 12, 709-712. [CrossRef] 